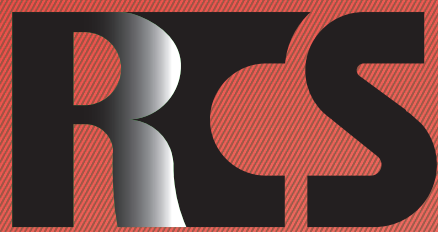

Depósito legal ppi $201502 Z U 4662$

Esta publicación científica en formato digital es continuidad de la revista impresa Depósito Legal: pp $197402 Z$ U789

- ISSN: 1315-9518 • ISSN-E: 2477-9431

Revista de Ciencias Sociales

Universidad del Zulia. Revista de la Facultad de Ciencias Económicas y Sociales Vol. XXVII,

No. 4,2021

Revista d

Sociales

Esta publicación científica en formato digital es continuidad de la revista impresa Depósito Legal: pp $197402 Z 1789$ ISSN: 1315-9518 


\title{
Estrategias empresariales agroecológicas en la Provincia del Azuay-Ecuador
}

\author{
Cantos Ochoa, Marcos Eduardo* \\ Guzmán Ávila, José Alciviades** \\ Ordóñez Espinosa, Cristina Guadalupe ${ }^{* * *}$ \\ Trelles Vicuña, Diego Fernando ${ }^{* * * *}$
}

\begin{abstract}
Resumen
Circunstancias adversas ha experimentado la sociedad ecuatoriana ante una crisis sanitaria y económica que ha obligado a las organizaciones agroecológicas a re-direccionar sus acciones para poder responder a los cambios del entorno nacional y contribuir con la seguridad alimentaria en Ecuador. Ante esto, el objetivo del presente trabajo fue determinar las estrategias de las asociaciones agroecológicas en la Provincia del Azuay en la Republica del Ecuador en el año 2020. Desde el empirismo, se llevó a cabo un diseño de investigación de campo, para indagar las estrategias que implementaron las mencionadas organizaciones y enfrentar las complejidades socioeconómicas; para ello se aplicó a 116 asociaciones un cuestionario estructurado a propietarios y encargados de las mismas. Entre los resultados encontrados predomina la orientación al mercado local desde la solidaridad y la ecología. Se concluye, que las asociaciones agroecológicas desarrollaron estrategias empresariales en una época hostil y desde un entorno nacional complejo, que condujo a crear acciones para estimular las ventas de productos y animales, imponiéndose las estrategias de crecimiento y competitividad.
\end{abstract}

Palabras clave: Agroecología; seguridad alimentaria; estrategas empresariales; crecimiento; competitividad.

Candidato a Doctor en Ciencias Sociales mención Gerencia en la Universidad del Zulia, Venezuela. Magister en Auditoria Integral. Ingeniero Empresarial. Docente de la unidad académica de Administración en la Universidad Católica de Cuenca, Ecuador. E-mail: mecantoso@ucacue.edu.ec (iD) ORCID: https://orcid.org/0000-0002-3340-5085

** $\quad$ Master en Desarrollo Emprendedor e Innovación. Especialista en Docencia Universitaria. Diploma Superior en Gerencia de Gobiernos Seccionales. Economista. Docente Tiempo Completo de la Unidad Académica de Administración en la Universidad Católica de Cuenca, Ecuador. E-mail: jguzman@. ucacue.edu.ec (iD ORCID: https://orcid.org/0000-0002-1558-8826

**** Candidata a Doctora en Ciencias Sociales mención Gerencia por la Universidad del Zulia, Venezuela. Magíster en Gestión Empresarial. Diplomado en Educación Superior por Competencias. Ingeniera Empresarial. Docente de la unidad académica de Administración en la Universidad Católica de Cuenca, Ecuador. E-mail: cgordoneze@ucacue.edu.ec (iD ORCID: https://orcid.org/0000-0001-5781-7781

***** Doctor en Jurisprudencia. Master en Administración de Empresas. Master en Derecho Constitucional. Docente Tiempo Completo de la Maestría en Derecho Constitucional con mención en Derecho Procesal Constitucional, Jefaturas de postgrado en la Universidad Católica de Cuenca, Ecuador. E-mail: dtrelles@ucacue.edu.ec (iD) ORCID: https://orcid.org/0000-0002-8466-7165

Recibido: 2021-06-05 · Aceptado: 2021-08-23 


\title{
Agroecological business strategies in the Province of Azuay-Ecuador
}

\begin{abstract}
Adverse circumstances Ecuadorian society has experienced in the face of a health and economic crisis that has forced agroecological organizations to redirect their actions in order to respond to changes in the national environment and contribute to food security in Ecuador. Given this, the objective of this work was to determine the strategies of agroecological associations in the Province of Azuay in the Republic of Ecuador in 2020. From empiricism, a field research design was carried out, to investigate the strategies who implemented the aforementioned organizations and face the socioeconomic complexities; For this, a structured questionnaire was applied to 116 associations for their owners and managers. Among the results found, the orientation to the local market from solidarity and ecology predominates. It is concluded that agroecological associations developed business strategies in a hostile time and from a complex national environment, which led to the creation of actions to stimulate the sales of products and animals, imposing growth and competitiveness strategies.
\end{abstract}

Keywords: Agroecology; food security; business strategists; growth; competitiveness.

\section{Introducción}

Señala la Comisión Económica para América Latina y el Caribe (CEPAL, 2020) que la propagación del COVID-19 desde mediados de marzo del año 2020 conllevó a los gobiernos de la región a implementar medidas sociales y de salud, para prevenir la propagación del virus y poder enfrentar las vicisitudes que aqueja a la población contagiada, ello también condujo a suspender temporalmente actividades económicas, así como limitar el desplazamiento de las personas entre regiones, locales y países, afectando la seguridad alimentaria en Latinoamérica, la cual "existe cuando todas las personas tienen acceso físico y económico a suficientes alimentos nutritivos para satisfacer sus necesidades alimenticias a fin de llevar una vida activa y sana" (Asamblea Nacional de Ecuador, 2009, p.2).

Apesar que la CEPAL y la Organizacional Internacional del Trabajo (CEPAL y OIT, 2020) consideran que el sector agrícola, ganadero y silvícola tendrán un impacto bajomedio en la producción económica como resultado de esta pandemia, para el Comité de
Seguridad Alimentaria Mundial (CSA, 2020), "los impactos del COVID-19 en el suministro y la demanda de alimentos afectarán directa e indirectamente a los cuatro pilares de la seguridad alimentaria y la nutrición: disponibilidad, acceso, uso y estabilidad" (p.9). Por lo que, aunado a los problemas de salud generados por la pandemia, ha habido incidencias en los sistemas alimentarios y en la seguridad alimentaria, generando "impactos negativos en la progresión de la pandemia al debilitar los sistemas inmunes: la desnutrición, al influir en el estado del sistema inmunológico, reduce la capacidad de prevenir y combatir enfermedades, incluidas las infecciosas" (Comité de Seguridad Alimentaria Mundial, 2020, p.2).

Al respecto, Ecuador enfrenta consecuencias socioeconómicas difíciles por el Covid-19, pues las dificultades de salud llegaron en un contexto económico crítico, que de acuerdo al Banco Central de Ecuador (2020), ha hecho evidente la reducción de la inversión, disminución de las exportaciones de bienes y servicios, contracción del gasto de consumo final de los hogares y mengua en 
el gasto del gobierno, entre otros, reflejando en el 2020 "un decrecimiento interanual que se encuentra en un rango comprendido entre $-7,3 \%$ (USD 66.678 millones en valores constantes) y $-9,6 \%$ (USD 65.015 millones)" (p.1).

A pesar que las actividades de producción y comercio de alimentos fueron excluidas de la restricción de movilidad, la interrupción de actividades afectó al sector agropecuario, sobretodo a la comercialización de alimentos, con una consecuente pérdida de empleos y una reducción de los ingresos por familia. Uno de los grupos más vulnerables ha sido la agricultura familiar campesina e Indígena en donde:

El Ministerio de Agricultura y Ganadería, con asistencia técnica de la FAO, realizó una evaluación de impactos del COVID-19 en los medios de vida agrícolas, la seguridad alimentaria y la producción agrícola, cuyos resultados evidencian un importante nivel de afectación en los hogares agropecuarios sobre el nivel de sus ingresos durante la pandemia y las condiciones respecto a su seguridad alimentaria, que podrían deteriorarse debido a la incertidumbre con respecto al acceso de alimento y el consumo basado en una dieta con menor aporte nutricional. (Organización de las Naciones Unidas de la Alimentación y la Agricultura [FAO], 2020, p.1)

Para atender esta situación, se aprobó la Ley Orgánica de Apoyo Humanitario (Asamblea Nacional de Ecuador, 2020a) para combatir la crisis sanitaria derivada de la pandemia, con el objeto de "establecer medidas de apoyo humanitario, necesarias para enfrentar las consecuencias derivadas de la crisis sanitaria ocasionada por el COVID-19 (...) y que permitan fomentar la reactivación económica y productiva del Ecuador" (p.3).

En este marco, la Prefectura del Azuay (2020) colocó en práctica un plan para el fortalecimiento de la reactivación ecoproductiva de esa provincia, con el objeto de "(...) mitigar la emergencia sanitaria, desarrollando acciones enfocadas a resistir, cambiando y remodelando los paradigmas actuales de producción y relación entre los seres humanos, ambiente y la economía" (p.5); en el cual la agroecología ha tenido un rol protagónico por su vinculación directa con la seguridad alimentaria para el desarrollo de estilos de vida campesinos por encima de la producción agroindustrial y la participación en la economía de mercado, así como por su capacidad de adaptación al estado de alarma sanitaria, para poder continuar con su actividad principal: La producción y distribución de alimentos a escala local, a la vez que se "(...) protegerán, conservarán los ecosistemas y promoverán la recuperación, uso, conservación y desarrollo de la agrobiodiversidad y de los saberes ancestrales vinculados a ella" (Asamblea Nacional de Ecuador, 2009, p.3).

Al respecto, Vásquez et al. (2018) sostienen que: "La práctica de la agricultura tradicional está ligada a la conservación de la cultura, a la reproducción social, a dinámicas económicas locales y de los diferentes conocimientos como el ambiental" (p.26); en ese sentido, "consiente la transmisión de saberes, así como la preservación tanto de herramientas como semillas propias, contribuyendo esta agricultura a nivel familiar con la seguridad alimentaria de las poblaciones" (León et al., 2021, p.359); con lo cual resulta vital la elaboración de productos agroecológicos.

Coinciden Patel y Goodman (2020), en que la inestabilidad socio-ecológica impuesta por el COVID-19, así como sus efectos en la agricultura y en las cadenas de suministro de alimentos, generan preocupaciones sobre la escasez de los mismos y los elevados precios, lo cual condujo a prestar atención a las potencialidades de la agroecología para aumentar la prosperidad rural, asegurar los sistemas alimentarios locales, ayudar a los agricultores a ser más autosuficientes y resistente a los acontecimientos graves. Sin embargo, para que ello sea fructífero, es necesario ser "capaz de administrar ante la complejidad actual del Ecuador, afrontar la incertidumbre, al igual que utilizar la flexibilidad, y hasta la imaginación, a efectos de anticiparse a los cambios, salir de la crisis y de inadecuadas estructuras productivas" (Useche et al., 2021, p.6). 
Al respecto, indican Altieri y Nicholls (2020) que "la agroecología muestra un camino a seguir al proporcionar los principios sobre cómo administrar los sistemas agrícolas que mejor puedan resistir crisis futuras, ya sean brotes de plagas, pandemias, trastornos climáticos o crisis financieras" (p.887). Ante esto, se consideró imperioso determinar las estrategias empresariales implementadas por las asociaciones agroecológicas en la Provincia del Azuay del Ecuador en el año 2020, en pro de la reactivación económica de esa actividad productiva y de la región.

El objetivo de este trabajo fue desarrollado desde el empirismo, empleando el método científico desde un tipo de investigación descriptivo y un diseño de campo. En el trabajo de campo, se estipuló que la población finita la comprendieron 498 asociaciones agroecológicas activas en esa provincia (Gobierno Provincial de Azuay y AGROKAWSAY, 2020), a la cual se extrajo una muestra aleatoria simple conformada por 116 asociaciones. Desde esas entidades, propietarios, encargados y/o representantes, se consideraron agentes informantes y respondieron a un cuestionario estructurado con escala Likert, conformado por 93 items.
Dicho instrumento superó las pruebas de fiabilidad: La validación por contenido, mediante la revisión de expertos sobre el contenido, consistencia y redacción del cuestionario; así como la confiabilidad, mediante el coeficiente alfa de Cronbach (0.90), garantizando la fiabilidad del mismo. Superada la fase de recolección de datos, se procedió a tabular, organizar y presentar los mismos en los siguientes apartados.

\section{Impulso de la agroecología en Ecuador}

Históricamente, el sistema agrícola en Ecuador ha experimentado desequilibrios estructurales, que no han sido solventados por los gobiernos ni por los agricultores. Sobre esto, el Ministerio de Agricultura, Ganadería, Acuacultura y Pesca (2016) indica que "encontramos que aún subsisten los viejos problemas de exclusión e inequidad en el campo, y han aparecido nuevos desafíos que se identifican en cuatro grandes campos de preocupación" (p.153), los cuales se presentan sus características en el Cuadro 1.

\section{Cuadro 1 \\ Problemas Estructurales de la Agricultura en Ecuador}

\begin{tabular}{|c|c|}
\hline Problema Agrícola & Características Estructurales \\
\hline $\begin{array}{l}\text { Obstáculos en el acceso a los } \\
\text { medios de producción esenciales }\end{array}$ & $\begin{array}{l}\text { - Desigual estructura y distribución en la tenencia de la tierra para uso } \\
\text { productivo. } \\
\text { - Microparcelamiento en la tenencia de la tierra dedicada a la agricultura. } \\
\text { - Inequidad en el acceso, distribución y gestión del riego parcelario. }\end{array}$ \\
\hline $\begin{array}{l}\text { Dificultades con el acceso } \\
\text { a los medios que potencian } \\
\text { los resultados de la actividad } \\
\text { agrícola. }\end{array}$ & $\begin{array}{l}\text { - Barreras en el acceso a los canales de comercialización y mercados. } \\
\text { - Insuficiente investigación e innovación y desarrollo tecnológico. } \\
\text { - Reducido desarrollo de los mercados de factores, productos e insumos para la } \\
\text { producción agropecuaria (crédito, insumos, asistencia técnica, etc.) }\end{array}$ \\
\hline $\begin{array}{l}\text { Gobernanza del desarrollo } \\
\text { territorial rural débil. }\end{array}$ & $\begin{array}{l}\text { - Entorno de políticas macroeconómicas y sectoriales desfavorables para el sector } \\
\text { agropecuario. } \\
\text { - Insuficiente prioridad fiscal al financiamiento para el sector agrícola. } \\
\text { - Institucionalidad pública y privada débil. }\end{array}$ \\
\hline $\begin{array}{l}\text { Deficiencia de las políticas } \\
\text { agropecuarias aplicadas }\end{array}$ & $\begin{array}{l}\text { - Persistencia de la pobreza en zonas rurales. } \\
\text { - Amplificación de los riesgos para la seguridad alimentaria de la población. } \\
\text { - Barreras para el desarrollo territorial rural y el crecimiento económico global. }\end{array}$ \\
\hline
\end{tabular}

Fuente: Ministerio de Agricultura, Ganadería, Acuacultura y Pesca (2016). 
Así como los problemas en esta actividad han sido históricos y han tenido que ser enfrentados generacionalmente por las familias campesinas, trabajadores del campo y asociaciones agrícolas, también los han enfrentado en la agroecología en Ecuador, pues también proviene de los sistemas agrícolas ancestrales (Chakra andina, Finca de los Pastos, Huerta de los Paltas, entre otros), y por tanto, han afrontado esas adversidades, a la vez que procuran mantener el medio ambiente productivo, equilibrado y económicamente viable, mediante principios ecológicos (Altieri, 1996), que se encuentran vigentes en los procesos de cultivos en la actualidad, así como han generado importantes aportes para la construcción de nuevos modelos agrarios (Cevallos, Urdaneta y Jaimes, 2019). Al respecto, señalan Intriago y Gortaire (2018) que la:

(...) dinamización del movimiento agroecológico y su perspectiva multidimensional en el marco de la Soberanía Alimentaria se amplifica y difunde la propuesta agroecológica, ahora con su enfoque integral y multidimensional, que (...) integra el valor del trabajo campesino de forma autónoma, logra autosatisfacción de necesidad alimentaria y mercados alternativos y rompe con la dependencia tecnológica. (p.98)

Con el impulso de movimientos campesinos y sociales en participaciones políticas, se fue ganando terreno en la defensa legal de la agroecología en el Ecuador, y con ello se impulsó el crecimiento de mercados, apertura a los productos agroecológicos en pequeños circuitos de comercialización, así como consolidación de redes y asociaciones agroecológicas en varias provincias del país. Esos avances promovieron la conformación de la normativa legal desde el año 2003, cuando ocurren dos sucesos importantes, a saber:

a. Aprobación del decreto 3609 (Ministerio de Agricultura y Ganadería [MAG], 2011), con el cual se regula la Producción Orgánica en Ecuador, y

b. Aprobación del Reglamento de la Normativa de la Producción Orgánica Agropecuaria, mediante el acuerdo Ministerial 177 (Ministerio de Agricultura y Ganadería [MAG], 2003), para la producción agroecológica, ecológica y biológica, empleando esos términos como sinónimos de orgánico, la cual fue reformulada en el año 2006.

Desde entonces se ha venido fortaleciendo el fundamento legal y aunque no es objeto de estudio de este trabajo, se hizo una síntesis cronológica de la normativa más relevante sobre la agroecología, que se puede observar en la Figura I, con la cual se entiende la relevancia de esta actividad en Ecuador, sobre todo por ser un país que su economía se dedica principalmente al desarrollo de la actividad primaria. 


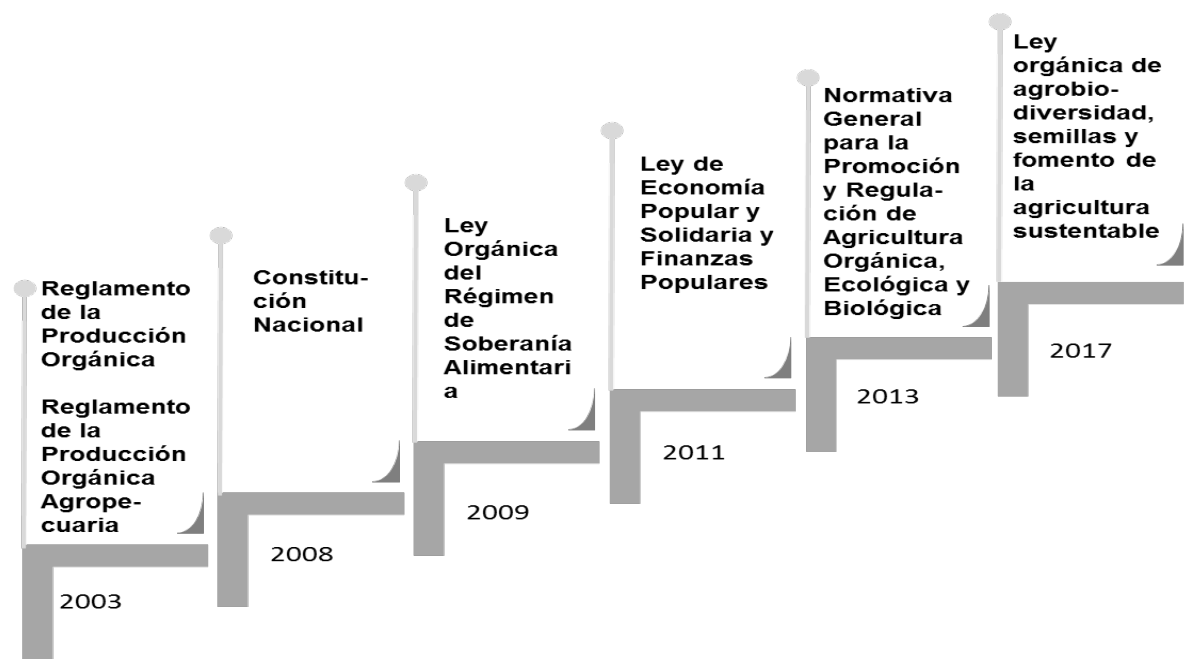

Fuente: Elaboración propia, 2021.

\section{Figura I: Cronología de la Normativa Legal de la Agroecología en Ecuador}

En el año 2008, con la Constitución Nacional (Asamblea Constituyente de Ecuador, 2011) se emite el apalancamiento a la agricultura a pequeña escala, los artículos 13 y 14 hace referencia a la agricultura sustentable y se "declara de interés público la preservación del ambiente, la conservación de los ecosistemas, la biodiversidad y la integridad del patrimonio genético del país, la prevención del daño ambiental y la recuperación de los espacios naturales degradados" (p.13). Asimismo, en el artículo 281, se reconoce a la soberanía alimentaria como objetivo nacional estratégico, lo cual dio bases para que en el año 2009 se crease la Ley Orgánica del Régimen de Soberanía Alimentaria (LORSA) (Asamblea Nacional de Ecuador, 2009).

Mediante esta ley, el Estado se compromete a coadyuvar a afianzar las redes de productores, consumidores y comerciantes entre territorios urbanos y rurales desde agrobiodiversidad (artículo 7-8), y de acuerdo con el artículo 14, a estimular "la producción agroecológica, orgánica y sustentable, a través de mecanismos de fomento, programas de capacitación, líneas especiales de crédito y mecanismos de comercialización en el mercado interno y externo" (Asamblea Nacional de Ecuador, 2009, p.10). Sin embargo, su puesta en marcha requirió de un conjunto de leyes relacionadas directamente a ella, como: Ley de tierras, ley de agro-biodiversidad y semillas, ley de comunas, ley de territorios, ley de desarrollo agrario, ley de agroindustria y empleo rural, ley de sanidad animal y vegetal, ley de acceso de los campesinos e indígenas al crédito público, entre otras.

$\mathrm{Si}$ bien es cierto que las leyes antes mencionadas son importantes en esta actividad, es también necesario mencionar que la Ley de Economía Popular y Solidaria (Asamblea Nacional de Ecuador, 2011), estimula el comercio justo y el consumo responsable (artículo 4), fortaleciendo los esfuerzos, así como avances a la agroecología, pues una sociedad solidaria se construye desde relaciones económicas inclusivas, equitativas, cuyo fin no es la acumulación de riqueza, 
sino satisfacer necesidades legítimas de las personas que respetan los límites naturales del ecosistema.

De manera complementaria, en el año 2013 se creó la Normativa General para la Promoción y Regulación de Agricultura Orgánica, Ecológica y Biológica, la cual propone la propiedad pública de las semillas y la agroecología como mecanismo para conservar la agrobiodiversidad (artículo 15); así como elevar la competitividad sectorial, proteger la salud de los consumidores, preservar el dinamismo vital del ambiente y mejorar el desarrollo de la agricultura ecológica, entre otros (Agencia Ecuatoriana de Aseguramiento de la Calidad del Agro [AGROCALIDAD], 2013).

También en el año 2013, se publicó la Ordenanza de Fomento Agroecológico del Gobierno de Pichincha, y en el año 2017, la Ley Orgánica de Agrobiodiversidad, Semillas y Fomento de la Agricultura Sustentable (Asamblea Nacional de Ecuador, 2017), tiene su impulso desde los planes Nacionales de Desarrollo Buen Vivir (20132017) (Secretaría Nacional de Planificación y Desarrollo [SENPLADES], 2013); y Toda una Vida (2017-2021) (SENPLADES, 2017), los cuales en el ámbito de la producción orientan la realización del Sumak Kawsay, impulsando el empleo de tecnologías ancestrales y revalorizadas para la producción de alimentos adecuados para garantizar la salud de la tierra, los cultivos y animales, en un contexto de respeto a la naturaleza; y en torno a ello, han surgido una amplia gama de códigos, reglamentos, leyes, entre otros, que coadyuvan al desenvolvimiento de esta actividad económica.

Desde este marco regulatorio, se impulsa la agrobiodiversidad en los modos de producción familiares campesinas, para el desarrollo económico y abastecimiento alimentario de los ecuatorianos con productos tradicionales y demanda de fuentes de empleo en diferentes territorios del Ecuador. Sin embargo, para Enríquez (2015), "no basta con la creación de direcciones, sino de fortalecerlas y dotarlas con capacidad de gestión territorial, recursos administrativos y financieros, para poder cumplir con los objetivos superiores de manera técnica y práctica" (p.182); de allí que, el acompañamiento gubernamental juega un rol importante para impulsar las estrategias empresariales que desarrollan las asociaciones agroecológicas, en procura de elevar valor agregado a sus productos y generar ventajas comparativas.

\section{Estrategias empresariales agroecológica en el Azuay ecuatoriano}

Se entienden a las estrategias empresariales como el conjunto de "acciones que despliegan las organizaciones, relacionadas directamente con los intereses y recursos organizacionales, y con las tendencias del segmento de mercado donde se desenvuelven" (Useche et al., 2020, p.72), configurando ideas de influencias clave sobre el bienestar presente y futuro de las organizaciones, lo cual implica abordar variables técnico-económicas, así como la vinculación del entorno, los recursos de la organización y los intereses de los grupos de interés.

Extenso ha sido el abordaje sobre este tema y diferentes vertientes en las ciencias económicas y sociales han surgido entre los autores, que han ahondado sobre sus concepciones, objetivos, alcances, con grandes aportes y disertaciones, sus estructuras, direccionamiento, clasificaciones, escuelas, enfoques, entre los cuales se pueden mencionar a Chandler (1962); Ansoff (1965); Miles y Snow (1978); Porter (1980); Miller (1986); Mintzberg (1987); Mintzberg, Ahlstrand y Lampel (1998); Camacho (2002); Whittington (2003); Furrer, Thomas y Goussevskaia (2008); Kotler y Keller (2016); entre otros.

Explican Pérez y Massoni (2009), que la teoría general de estrategias ha hecho importantes aportes a la teoría de los recursos, a la teoría de las capacidades, de las competencias, en la cadena de valor, en la gestión del conocimiento, entre otros. Asimismo, se han creado una diversidad de clasificaciones de estrategias empresariales, 
unas relacionando las empresas con la capacidad de adaptación al entorno y su orientación estratégica (Miles y Snow, 1978); y otras clasificaciones por niveles (Hofer y Schendel, 1978); entre otras. También esta temática se ha insertado en los enfoques organizacionales, centrando sus aportes en las funciones estratégicas (Barnard, 1938; Simon, 1944; Senge, 2005).

Por otra parte, desde enfoques ortodoxos orientados a la rivalidad, al marketing y ventaja competitiva, se encuentran Ansoff (1965); y, Porter (1979; 1980; 1985; 1998); también Hamel y Prahalad (1989); Munuera y Rodríguez (2007); así como Kotler y Keller (2016), quienes han hecho contribuciones en innovación de nuevos espacios y posiciones en el mercado que reivindican las habilidades y competencias distintivas frente a los competidores; mientras que Mintzberg (1987); construyó diez escuelas con enfoques múltiples en ese campo, abriendo un abanico de oportunidades para estudiar las orientaciones, decisiones y tendencias estratégicas.

$$
\text { Para abordar las estrategias }
$$
empresariales, resulta clave identificar el direccionamiento estratégico y con ello, la combinación e integración de acciones que en su conjunto conforman un marco de acción, con el cual los gobiernos provinciales impulsan la reactivación económica en cada región del Ecuador, y coadyuvan a que las asociaciones tengan acceso a servicios básicos, recursos, materiales, equipos e insumos, para cultivar la cantidad y variedad de productos necesarios que puedan cubrir la demanda nacional; sin embargo, son las asociaciones quienes tienen la responsabilidad de trabajar la tierra, cultivar y criar animales, sin afectar a la biodiversidad del campo y del medio ambiente en general, e ir implementando las estrategias empresariales acorde a su actividad económica y entorno.

Ante esto, en el presente trabajo se indagaron las estrategias empresariales ortodoxas, pues como se indicó anteriormente se está en el marco de reactivación agroecológica y ello requiere de estrategias tanto de crecimiento como competitivas desde la mezcla de mercadeo, para lo cual, se examinaron las acciones implementadas en los productos, precio, distribución y comunicación, porque indican los cursos de acción, de acuerdo con los medios y recursos disponibles de las asociaciones, las cuales se presentan en el siguiente apartado.

\section{Estrategias empresariales en la actividad agroecológica}

Al recopilar la información se encontró que la actividad agroecológica se mantuvo activa todo el año, predominando las asociaciones comunitarias (72,03\%) en esa actividad en los cantones de Nabon $(35,59 \%)$ y Cuenca $(33,05)$, y el resto de las zonas geográficas de la mencionada actividad $(31,26 \%)$ están distribuidas en otros cantones como: Sigsi, Gualaceo, Ponce Enríquez, El Pan, Santa Isabel, entre otros. En esa actividad, se detectó mayor presencia de las mujeres (0-40 años) como fuerza laboral (88,14\%); asimismo, los hombres (71,19\%) entre edades de 0 a 40 años, también juegan un rol importante en la cría de animales y cultivos de la tierra para producir suficientes alimentos, pues el 93,22\% de los encuestados considera que pueden garantizar la soberanía alimentaria, a través de la implementación de prácticas agroecológicas.

A pesar que su devenir se encontraba en un entorno turbulento, caracterizado por una crisis económica estructural y por una crisis sanitaria, producto del coronavirus COVID-19, también ha habido presión por parte de la sociedad para que haya garantía y soberanía alimentaria; dichos aspectos han hecho cuesta arriba las gestiones y operaciones, convirtiendo en un reto salir airosos ante tan complejo contexto, sobre todo en esos momentos para toda la población. Sin embargo, la agroecología en Ecuador no se detuvo, aunque fue necesario reconsiderar sus estrategias, dado que algunas de ellas perdieron pertinencia ante la realidad compleja, por lo que resultó imperioso reorientar su direccionamiento estratégico para adaptarse al entorno, y responder a las nuevas necesidades 
de la economía nacional.

En tal sentido, reconocen las asociaciones agroecológicas que por sí solas no es posible producir la cantidad de productos suficientes para cubrir la demanda nacional y exportar, para este último fin, requieren apoyo por parte del gobierno para crear cadenas cortas de suministro de alimentos, esquemas de mercadeo directo, mercadeo cooperativo, mercados de agricultores, huertos comunitarios y escolares, sistemas de intercambio, consumo directo en la finca, así como un sistema de financiamiento público eficaz para la agroecología $(95,75 \%)$, lo cual no es una tarea fácil de realizar por parte del gobierno, a pesar de la creación de la Ley de Ordenamiento de las Finanzas Públicas (Asamblea Nacional de Ecuador, 2020b), pues:

Las necesidades brutas de financiamiento que enfrenta Ecuador para 2020 representarían cerca de USD $13 \quad 400$ millones, con una brecha de financiamiento aún sin cubrir de al menos USD 2000 millones (FMI, 2020b; MEF, 2020a). En este contexto, las principales opciones en que ha estado avanzando el país han sido: 1) financiamiento externo adicional; 2) reestructuración de la deuda externa; 3 ) reordenar los recursos disponibles en el plano doméstico, reorientando el gasto hacia acciones prioritarias. (Organización para la Cooperación y Desarrollo Económicos [OCDE], 2020, p.7)

Tomando en consideración las limitaciones financieras, sanitarias y socioeconómicas del país, las asociaciones consideraron continuar su devenir estratégico hacia la participación en el mercado. Al respecto, se encontró que las estrategias de crecimiento fueron las menos predominantes, solo un $16,10 \%$ manifestó haber crecido, es decir, que no hubo aumento en las ventas, por lo que se mantuvo estable la participación de mercado, ese bajo porcentaje indica según Ruiz (2018), un efecto negativo en la vitalidad de las empresas, desestimula las iniciativas y aumenta la desmotivación de las personas que trabajan la tierra y cría los animales.

Lo anterior, es coherente con las circunstancias de represión económica nacional, a pesar de contar con terrenos para ampliar la producción $(66,10 \%)$, y estar dispuestos a ampliar la inversión en los cultivos $(83,90 \%)$, no cuentan con las posibilidades de implementar estrategias relacionadas a innovaciones $(77,075 \%)$, tampoco tienen recursos económicos para adquirir maquinaria $(88,08 \%)$, ni tecnificar procesos agrícolas $(55,08 \%)$, entre otros; con lo cual, no se pueden generar "modificaciones internas, ni aumento de las dimensiones y cambios en las características de los objetos sometidos a tal proceso" (Aguilera, 2010, p.95-96).

Por otra parte, se encontró de manera predominante la implementación de estrategias competitivas, mediante acciones en la mezcla de mercadeo, a saber: Producto, precio, distribución y comunicación, que en su conjunto de acuerdo a Porter (1980; 1985), se implementan para competir y alcanzar ventajas sostenibles a largo plazo, y en este trabajo, está enfocada en el manejo ancestral, así como ecológico de los recursos naturales en los cultivos y cría de animales, como se explicó anteriormente.

En esta actividad los cultivos han sido diversificados, así como la cría de animales para el consumo de los seres humanos (ver Tabla 1), pero los elementos diferenciadores de los mismos radican en que se emplean fertilizantes orgánicos $(66,01 \%)$, abono orgánico $(77,50 \%)$, previenen las plagas $(87,06 \%)$, tienen plantas sanas, resistentes a plagas $(82,75 \%)$, entre otros aspectos, $y$ al seguir procedimientos ancestrales para conservar los nutrientes en la tierra y el agua, sin degradar el medio ambiente, obtienen productos con mayores propiedades, y por tanto, más saludables para los seres humanos, esos elementos de diferenciación, de acuerdo a Porter (1980), buscan generar ventajas competitivas en el mercado. 


\section{Tabla 1 \\ Productos Cultivados y Animales Criados}

\begin{tabular}{lc}
\hline \multicolumn{1}{c}{ Productos } & Porcentaje \\
\hline Hortalizas & $54 \%$ \\
Verduras & $41 \%$ \\
Frutas y derivados & $34 \%$ \\
Cereales cocinados y tiernos & $28 \%$ \\
Lácteos & $26 \%$ \\
Tubérculos & $23 \%$ \\
Cría de animales menores & $24 \%$ \\
\hline
\end{tabular}

Fuente: Elaboración propia, 2021.

Resulta importante destacar que, como parte de la práctica ecológica en la siembra de estos productos, el $88,79 \%$ de los agentes informantes expresaron que cultivan varias especies, evitando los monocultivos y la degradación de los nutrientes en la tierra, siendo "una expresión de la implementación de las estrategias del uso múltiple, que responde a una racionalidad tanto ecológica, sociocultural y en la actualidad a la económica" (Loyola, 2016, p.3).

En ese marco, también manifestaron que consideran el suelo como un ser vivo $(77,58 \%)$, y para ello, emplean un alto reciclaje de material orgánico $(80,17 \%)$, provenientes de residuos de la cosecha; esas prácticas con el tiempo han permitido tener capacidad de recuperarse ante cambios climáticos (62,93\%), así como mantener estables la cantidad y las formas de producción (50,86\%). Estos resultados corroboran que los "cultivos asociados o múltiples, maximizan las interacciones agroecológicas, ya que el agrupamiento de plantas con distinta eficiencia energética, hábitos de crecimiento y estructuras radiculares, emplean de forma más eficiente la energía solar, nutrientes y agua" (DamiánHuato et al., 2010, p.71).

Con respecto al precio, no fueron significativas las estrategias desarrolladas, porque los problemas estructurales de la economía nacional, aunado a altos niveles de desempleo y un mercado laboral inestable, dificultó que las personas tuvieran preferencias, así como apreciaran el valor de los productos ecológicos; por lo que, el precio de acuerdo a las cualidades agroecológicas no fue predominante y no tuvo incidencia competitiva sobre estos productos; siendo la orientación del mercado el criterio para establecer el precio $(82,20 \%)$, considerando que desde este criterio se sobrepone según Fuentes (2010):

Una capacidad distintiva que parte desde el exterior de la firma hacia el interior y conecta a la empresa con el entorno a través de la generación de sensibilidad con el mercado, las relaciones con sus clientes y los lazos con el canal de distribución. (p.27)

Siguiendo en el marco del mercado, $94,92 \%$ de las asociaciones consideran que necesitan nuevas maneras de distribuir los productos, así como mejorar la infraestructura para facilitar la distribución de los productos, y $94,92 \%$ afirma que para que haya el crecimiento de las asociaciones se necesitan nuevos canales de distribución, así como apoyo del gobierno provincial (94,92\%), para construir nuevas formas de distribuir los productos, pues ello coadyuvará a que la población tenga más alcance a los mismos, procurando los precios justos.

Sin embargo, se debe tener cuidado con este aspecto, pues por lo general ampliar los canales de distribución implica incremento de los costos, que se trasladan a los precios, es decir, un alza de los mismos, haciendo menos atractivo el consumo de productos ecológicos, por lo menos en época de recesión económica, y altos niveles de desempleo que conllevan a una menor capacidad adquisitiva. En tal sentido, Contreras, Paredes y Turbay (2017), consideran como una mejor estrategia de distribución aquella mediante circuitos cortos alternativos, pues son mediante:

(...) redes alternativas al mercado común en las que existen relaciones directas entre productores y consumidores, como: ferias campesinas, tiendas de productos campesinos, canastas de consumidores, compras públicas por parte del Estado y mercados internacionales de comercio justo, las cuales contribuyen a la soberanía alimentaria porque implican atención no solo al proceso de circulación de los productos sino a su producción y reproducción. (p.72)

Con este tipo de circuitos se impulsa 
la reducción de costos por transacciones, así como relaciones recíprocas y solidarias guiadas por una lógica de solidaridad, distinta al lucro porque disminuyen los costos de mantenimiento, así como reparación de transporte, dando posibilidad a mejorar los precios, lo cual es importante para la sostenibilidad de los circuitos, como también para fortalecer la soberanía alimentaria en las regiones, porque los productores y compradores obtienen más beneficios justos en la compra/venta de los productos.

En relación a las estrategias de comunicación, se consultó sobre el empleo del internet para informar y ampliar sus relaciones con otros productores y/o consumidores, pues en el año 2020, fue el medio más empleado para vincularse, así como potenciar negocios en todo el mundo. Al respecto, se encontró que el empleo de los medios de comunicación digitales no ha tenido fines comerciales, pues $82,20 \%$ no han vendido productos por internet, tampoco contactan nuevos distribuidores $(85,59 \%)$, y no lo utilizan para buscar nuevos proveedores $84,75 \%$, más allá de la motivación que puedan tener o la falta de información estos aspectos en internet, se adiciona que en las zonas rurales existen limitaciones de acceso a conectividad y equipamiento de tecnología, así como falta de conocimiento sobre plataformas virtuales.

Como ha podido observarse, existen varias estrategias para lograr ventajas competitivas, y su sostenimiento coadyuvará a que resistan a las acciones de la competencia, así como se superen barreras del entorno (Porter, 1998). Al respecto, el direccionamiento estratégico se ha basado en la diferenciación predominantemente y es claro que, "lo que se quiere es conseguir que el mercado acepte un producto o servicio superior a la competencia o tenga más demanda a igualdad de precio" (Castro, 2010, p.260), fundado en las características intrínsecas del producto en un sentido amplio, donde se consideren la calidad, los valores nutricionales, y el grado de exclusividad, entre otros.

\section{Conclusiones}

En el marco de los Objetivos de Desarrollo Sostenible (ODS) que entre ellos se procura erradicar el hambre e impulsar la preservación de la vida de los ecosistemas terrestres, el gobierno ecuatoriano ha venido haciendo aportes importantes; sin embargo, desde el año 2020 ese impulso se vio mermado ante la crisis económica y de salud que ha profundizado la pandemia del coronavirus COVID-19. A pesar del entorno turbulento, los gobiernos provinciales impulsaron la reactivación económica en cada región, estimulando a las asociaciones agroecológicas a cultivar y realizar todos los procesos que permitan generar cantidad, como también variedad de productos, necesarios para poder cubrir la demanda de productos en el ámbito nacional.

Sin embargo, el sector agroalimentario no ha estado exento al coronavirus COVID-19, cuyos efectos han interrumpido el normal funcionamiento de las cadenas de valor alimentarias, teniendo que enfrentar inéditos problemas que han dado cabida a nuevos desafíos; a pesar de ello, la producción de alimentos no se ha detenido y no podía ser de otra forma, porque son esenciales, así como el salario de los trabajadores, para cubrir las necesidades básicas de las familias ecuatorianas en esa provincia.

En un intento de paliar las limitaciones mencionadas, las asociaciones agroecológicas han adoptado un rol protagónico ante la sociedad, para trabajar la tierra, cultivar con los saberes ancestrales y criar animales, sin afectar la biodiversidad del campo e ir implementando estrategias empresariales de crecimiento y competitividad, acordes a su actividad económica y entorno para participar en su segmento de mercado de manera activa.

A pesar que las estrategias de crecimiento fueron débiles, dicha decisión se corresponde con la recesión estructural económica que enfrentan, pues confrontan grandes limitaciones, como: Dificultades de conectividad, que facilite información veraz, para ofertar sus productos y demandar 
insumos con los proveedores, obstáculos en el acceso a créditos para la reactivación económica de emprendimientos, y falta de apoyo gubernamental para la generación de nuevas iniciativas de comercialización, entre otros.

Menos tímidas que las estrategias de crecimiento fueron las estrategias competitivas, aunque las acciones de precio y comunicación fueron las más débiles, las primeras, conexas a la inestabilidad del mercado laboral que afecta la capacidad de los consumidores e incide desfavorablemente en el precio de los productos; y las segundas, porque problemas de acceso de internet y falta de conocimiento de las tecnología de información y comunicación para fines empresariales, como: Búsqueda con nuevos proveedores y nuevos mercados, no estimula el uso de dichos medios en la zona rural, para ampliar la comercialización o buscar mejores precios de los materiales, equipos y maquinaria que requieren para desarrollar la mencionada actividad.

Así que, ha sido notoria la importancia de las estrategias empresariales por parte de las asociaciones agroecológicas, para competir eficiente y solidariamente en los mercados, de ahí la importancia de poder tomar en cuenta todos los elementos estratégicos básicos para establecer una estrategia coherente y alcanzable en el corto plazo.

Elegir una estrategia agroecológica requiere de la integración de ideas y métodos desde varias disciplinas, lo cual exige salir del marco disciplinario agrícola, como en esta oportunidad se ha hecho un acercamiento desde las ciencias económicas y administrativas, con el fin de entender las estrategias que desarrollan las asociaciones en su segmento de mercado, porque es notorio que estas asociaciones también dependen de factores coordinados por una buena administración empresarial.

Siguen existiendo desafíos en un marco socioeconómico controversial para esta actividad; sin embargo, tanta responsabilidad tiene el gobierno como los ecuatorianos por continuar evitando la degradación de los hábitats y fortalecer la relación de los seres humanos con la naturaleza, desde el respeto y la protección de la misma, para que ella continúe protegiendo a la humanidad, siendo esa la gran estrategia.

\section{Referencias bibliográficas}

Agencia Ecuatoriana de Aseguramiento de la Calidad del Agro - AGROCALIDAD. (2013). Instructivo de la normativa general para promover y regular la producción orgánica - ecológica - biológica en el Ecuador. AGROCALIDAD. https://www. agrocalidad.gob.ec/wp-content/ uploads $/ 2020 / 05 /$ by $3 . p d f$

Aguilera, A. (2010). Direccionamiento estratégico y crecimiento empresarial: algunas reflexiones en torno a su relación. Pensamiento \& Gestión, (28), 85-106.

Altieri, M. A. (Ed.) (1996). Agroecology: The science of sustainable agriculture. Editorial Westview Press.

Altieri, M. A., y Nicholls, C. I. (2020). Agroecology and the reconstruction of a post-COVID-19 agriculture. The Journal of Peasant Studies, 47(5), 881-898. https://doi.org/10.1080/0306 $\underline{6150.2020 .1782891}$

Ansoff, H. I. (1965). Corporate strategy. McGraw-Hill Inc.

Asamblea Constituyente de Ecuador (2011). Constitución de la República del Ecuador 2008. https://www.oas.org/ juridico/pdfs/mesicic4 ecu const.pdf

Asamblea Nacional de Ecuador (2009). Ley Orgánica del Régimen de la Soberanía Alimentaria. Registro Oficial Suplemento 583 de 5 de Mayo del 2009. https://www.wipo.int/edocs/ lexdocs/laws/es/ec/ec046es.pdf

Asamblea Nacional de Ecuador (2011). Ley 
Orgánica de Economía Popular y Solidaria del Sistema Financiero. https://www.oas.org/juridico/pdfs/ mesicic4 ecu econ.pdf

Asamblea Nacional de Ecuador (2017). Ley Orgánica de Agrobiodiversidad, Semillas y Fomento de Agricultura Sustentable. Registro Oficial Suplemento Año I - $\mathrm{N}^{\mathrm{o}} 10$ Quito, jueves 8 de junio de 2017. https:// extwprlegs 1.fao.org/docs/pdf/ ecu168628.pdf

Asamblea Nacional de Ecuador (2020a). Ley Orgánica de Apoyo Humanitario. Registro Oficial Suplemento 229 de $22-$ jun.-2020. https://www.emov.gob.ec/ sites/default/files/transparencia_2020/ a2 41.pdf

Asamblea Nacional de Ecuador (2020b). Ley Orgánica para el Ordenamiento de las Finanzas Públicas. Suplemento del Registro Oficial No. 253. 24 de julio de 2020. http://www.pge.gob.ec/images/ documentos/LeyTransparencia/2021/ enero/a2/ley organica para el ordenamiento de las finanzas publicas.pdf

Banco Central de Ecuador (3 de junio de 2020). El COVID-19 pasa factura a la economía ecuatoriana: Decrecerá entre $7,6 \%$ y $9,6 \%$ en 2020. Banco Central de Ecuador. https://www.bce. fin.ec/index.php/boletines-de-prensaarchivo/item/1369-el-covid-19-pasafactura-a-la-economia-ecuatorianadecrecera-entre-7-3-y-9-6-en-2020

Barnard, C. (1938). The functions of the executive. Harvard University Press.

Camacho, M. (2002). Direccionamiento estratégico: Análisis de una herramienta poderosa. Revista Via Salud, 21(2), 125-137. https://oes. org.co/portfolio/revista-via-saludno21-2002/

Castro, E. (2010). Las estrategias competitivas y su importancia en la buena gestión de las empresas. Revista de Ciencias Económicas, 28(1), 247-276.

Cevallos, M., Urdaneta, F., y Jaimes, E. (2019). Desarrollo de sistemas de producción agroecológica: Dimensiones e indicadores para su estudio. Revista de Ciencias Sociales (Ve), XXV(3), 172185.

Chandler, A. D. (1962). Strategic and structure: Chapters in the history of the American industries enterprise. Beard Books.

Comisión Económica para América Latina y el Caribe - CEPAL (2020). Estudio Económico de América Latina y el Caribe: Principales condicionantes de las políticas fiscal y monetaria en la era pospandemia de COVID-19. CEPAL. https://www.cepal.org/ es/publicaciones/46070-estudioeconomico-america-latina-caribe2020-principales-condicionantes

Comisión Económica para América Latina y el Caribe y Organización Internacional del Trabajo - CEPAL y OIT (2020). Coyuntura Laboral en América Latina y el Caribe. El trabajo en tiempos de pandemia: Desafios frente a la enfermedad por coronavirus (COVID-19). CEPAL y OIT. https://www.cepal.org/es/ publicaciones/45557-coyunturalaboral-america-latina-caribe-trabajotiempos-pandemia-desafios-frente

Comité de Seguridad Alimentaria Mundial - CSA (2020). Documento interino de cuestiones sobre el: Impacto del COVID-19 en la seguridad alimentaria y la nutrición (SAN). CSA. https://www.fao.org/fileadmin/ templates/cfs/Docs1920/HLPE 2020/ New HLPE paper COVID ES.pdf

Contreras, J., Paredes, M., y Turbay, S. (2017). Circuitos cortos de comercialización agroecológica en 
Cantos Ochoa, Marcos Eduardo; Guzmán Ávila, José Alciviades; Ordóñez Espinosa, Cristina Guadalupe y Trelles Vicuña, Diego Fernando

Estrategias empresariales agroecológicas en la Provincia del Azuay-Ecuador

el Ecuador. IDESIA, 35(3), 71-80. http://dx.doi.org/10.4067/S0718$\underline{34292017005000302}$

Damián-Huato, M. A., Ramírez-Valverde, B., Aragón-García, A., HuertaLara, M., Sangerman-Jarquin, D. M. D. J., y Romero-Arenas, O. (2010). Manejo del maíz en el estado de Tlaxcala, México: Entre lo convencional y lo agroecológico. Revista Latinoamericana de Recursos Naturales, 6(2), 67-76.

Enríquez, F. (2015). Seguridad alimentaria: Responsabilidad de los Gobiernos Autónomos Descentralizados Provinciales. Ediciones Abya-Yala.

Fuentes, P. A. (2010). La orientación al mercado: Evolución y medición de un enfoque de gestión que trasciende al marketing. Perspectivas, (25), 25-83.

Furrer, O., Thomas, H., y Goussevskaia, A. (2008). The structure and evolution of the strategic management field: A content analysis of 26 years of strategic management research. International Journal of Management Reviews, 10(1), 1-23. https://doi.org/10.1111/ j.1468-2370.2007.00217.x

Gobierno Provincial de Azuay y AGROKAWSAY (2020). Listado de actores relacionados con la agroproducción 2020. República del Ecuador.

Hamel, G., y Prahalad, C. K. (1989). Strategic intent. Harvard Business Review, 89, 63-76.

Hofer, C. W., y Schendel, D. (1978). Strategy formulation: Analytical concepts. Editorial West Publishing Co.

Intriago, R., y Gortaire R. (2018). Agroecología en el ecuador. Proceso histórico, logros y desafíos. Agroecología, 11(2), 95-103. https://revistas.um.es/ agroecologia/article/view/330131
Kotler, P., y Keller, K. L. (2016). Dirección de marketing. Pearson Educación.

León, N., Pérez, G. S., Becerra, S., y Rodríguez, J. L. (2021). Revalorización y sostenibilidad tecnológica de los andenes interandinos agroecológicos en la región Arequipa-Perú. Revista de Ciencias Sociales (Ve), XXVII(1), 357-367. https://doi.org/10.31876/rcs. v27i1.35316

Loyola, J. G. (2016). Conocimientos y prácticas ancestrales y tradicionales fortalecen la sustentabilidad de los sistemas hortícolas de la parroquia de San Joaquín. LA GRANJA. Revista de Ciencias de la Vida, 24(2), 2942. https://doi.org/10.17163/lgr. n24.2016.03

Miles, R. E., y Snow, C. C. (1978). Organizational strategy, structure and process. McGraw-Hill.

Miller, D. (1986). Configurations of strategy and structure: Towards a synthesis. Strategic Management Journal, 7(3), 233-249.

Ministerio de Agricultura y Ganadería - MAG (2003). Acuerdo Ministerial 177. Reglamento de la normativa para la producción orgánica agropecuaria en el Ecuador. Registro Oficial Edición 154. 25/VIII/2003. https:// www.ecolex.org/es/details/legislation/ acuerdo-no-177-reglamento-de-lanormativa-de-la-produccion-organicaagropecuaria-en-el-ecuador-lexfaoc040974/

Ministerio de Agricultura y Ganadería - MAG (2011). Decreto Ejecutivo 3609. Normativa General para promover y regular la producción orgánica en el Ecuador. Texto Unificado de Legislación Secundaria del MAG, LIBRO I. http://extwprlegs1.fao.org/ docs/pdf/ecu165583.pdf

Ministerio de Agricultura, Ganadería, 
Acuacultura y Pesca (2016). La política agropecuaria ecuatoriana: Hacia el desarrollo territorial rural sostenible 2015-2025 I Parte. http://extwprlegs1. fao.org/docs/pdf/ecu183434.pdf

Mintzberg, H. (1987). Crafting strategy. Harvard Business Review. https://hbr.org/1987/07/craftingstrategy?language $=\mathrm{es}$

Mintzberg, H., Ahlstrand, B., y Lampel, J. (1998). Strategy safari: A guided tour through the wilds of strategic management. The Free Press.

Munuera, J. L., y Rodríguez, A. I. (2007). Estrategias de marketing: Un enfoque basado en el proceso de dirección. ESIC Editorial.

Organización de las Naciones Unidas de la Alimentación y la Agricultura - FAO (30 de octubre de 2020). El rol de los gobiernos locales en los procesos de recuperación económica en el sector agropecuario en un escenario de pandemia. FAO. https://www.fao. org/ecuador/noticias/detail-events/ es/c/1320388/

Organización para la Cooperación y Desarrollo Económicos - OCDE (2020). Impacto financiero del Covid-19 en Ecuador: Desafios y respuesta. OCDE. https://www.oecd.org/dev/Impactofinanciero-COVID-19-Ecuador.pdf

Patel, R., y Goodman, J. (2020). The long new deal. The Journal of Peasant Studies, 47(3), 431-463. https://doi.org/10.108 $\underline{0 / 03066150.2020 .1741551}$

Pérez, R. A., y Massoni, S. (2009). Hacia una teoría general de la estrategia: El cambio de paradigma en el comportamiento humano, sociedad y las instituciones. Editorial Ariel.

Porter, M. E. (1979). How competitive forces shaped strategy. Harvard Business Review, 57(2), 137-145.
Porter, M. E. (1980). Competitive strategy. Techniques for analyzing industries and competitors. The Free Press.

Porter, M. E. (1985). Competitive advantage. Free Press.

Porter, M. E. (1998). The competitive advantage of nations. Free Press.

Prefectura de Azuay (2020). Plan para el fortalecimiento de la reactivación eco-productiva de la provincia del Azuay. Gobierno Provincial de Azuay, Republica de Ecuador.

Ruiz, A. (2018). La estrategia de crecimiento intensivo de desarrollo de productos: Conceptos, uso y aplicación. https://www.researchgate.net/ publication/326815962 La estrategia de crecimiento intensivo de desarrollo de productos conceptos uso y aplicacion

Secretaría Nacional de Planificación y Desarrollo-SENPLADES(2013).Plan Nacional del Buen Vivir 2013-2017. Todo el mundo mejor. SENPLADES. https://observatorioplanificacion. cepal.org/sites/default/files/plan/files/ Ecuador\%20Plan\%20Nacional\%20 del $\% 20$ Buen $\% 20$ Vivir.pdf

Secretaría Nacional de Planificación y Desarrollo - SENPLADES (2017). Plan Nacional de Desarrollo 20172021. Toda una Vida. SENPLADES. https://observatorioplanificacion. cepal.org/sites/default/files/plan/files/ EcuandorPlanNacionalTodaUnaVi da20172021.pdf

Senge, P. (2005). La quinta disciplina. Ediciones Juan Granica, S.A.

Simon, H. (1944). Decision-making and administrative strategic management. Public Administration Review, 4, 1630.

Useche, M., Salazar, F., Barragán, C., y Sánchez, P. (2020). Horizontes 
Cantos Ochoa, Marcos Eduardo; Guzmán Ávila, José Alciviades; Ordóñez Espinosa, Cristina Guadalupe y Trelles Vicuña, Diego Fernando

Estrategias empresariales agroecológicas en la Provincia del Azuay-Ecuador

estratégicos empresariales en América Latina ante la pandemia generada por la COVID-19. SUMMA. Revista disciplinaria en ciencias económicas $y$ sociales, 2(E), 59-86. https://doi. org/10.47666/summa.2.esp. 07

Useche, M. C., Vásquez, L. M., Salazar, F. I., y Ordóñez Gavilanes, M. (2021). Fórmula estratégica empresarial para pymes en Ecuador ante la covid-19. Revista Universidad \&
Empresa, 23(40), 1-22. https://doi. org/10.12804/revistas.urosario.edu.co/ empresa/a.9309

Vásquez, A. Y., Chávez, C., Herrera, F., y Carreño, F. (2018). Milpa y seguridad alimentaria: El caso de San Pedro El Alto, México. Revista de Ciencias Sociales (Ve), XXIV(2), 24-36.

Whittington, R. (2003). ¿Qué es la estrategia? ¿Realmente importa? Tompson Editores. 